ARTICLE

\title{
A Drosophila RNAi library modulates Hippo pathway-dependent tissue growth
}

Joseph H.A. Vissers ${ }^{1,2}$, Samuel A. Manning ${ }^{1,3}$, Aishwarya Kulkarni ${ }^{1}$ \& Kieran F. Harvey ${ }^{1,2,3}$

Libraries of transgenic Drosophila melanogaster carrying RNA interference (RNAi) constructs have been used extensively to perform large-scale functional genetic screens in vivo. For example, RNAi screens have facilitated the discovery of multiple components of the Hippo pathway, an evolutionarily conserved growth-regulatory network. Here we investigate an important technical limitation with the widely used VDRC KK RNAi collection. We find that approximately $25 \%$ of VDRC KK RNAi lines cause false-positive enhancement of the Hippo pathway, owing to ectopic expression of the Tiptop transcription factor. Of relevance to the broader Drosophila community, ectopic tiptop (tio) expression can also cause organ malformations and mask phenotypes such as organ overgrowth. To enhance the use of the VDRC KK RNAi library, we have generated a D. melanogaster strain that will allow researchers to test, in a single cross, whether their genetic screen of interest will be affected by ectopic tio expression.

\footnotetext{
${ }^{1}$ Cell Growth and Proliferation Laboratory, Peter MacCallum Cancer Centre, 7 St Andrews Place, East Melbourne, Melbourne, Victoria 3002, Australia. ${ }^{2}$ Sir Peter MacCallum Department of Oncology, University of Melbourne, Parkville, Victoria 3010, Australia. ${ }^{3}$ Department of Pathology, University of Melbourne, Parkville, Victoria 3010, Australia. Correspondence and requests for materials should be addressed to J.H.A.V. (email: joep.vissers@petermac.org) or to K.F.H. (email: kieran.harvey@petermac.org).
} 
G enetic screens using the vinegar fly, Drosophila melanogaster, have been essential for our understanding of metazoan development. The generation of libraries of transgenic flies carrying inducible RNA interference (RNAi) vectors has enabled high-throughput functional genetic screens in vivo ${ }^{1}$. This technology has been successfully applied to uncover new regulators of a diverse range of biological functions ${ }^{2,3}$.

The VDRC KK collection is an RNAi library that is used in Drosophila laboratories globally (www.vdrc.at). It was designed to feature site-specific integration of hairpin RNAs carried in a transgenic vector at a defined locus (40D on chromosome $2 \mathrm{~L}$ ), to ensure consistent expression of different RNA hairpins and to alleviate the potential for disruption or misexpression of host genes. However, a recent report showed that only a subset of VDRC KK RNAi lines actually carry a transgene at 40D, whereas all lines possess a transgene at a secondary site that was unintentionally targeted ( $30 \mathrm{~B}$ on chromosome $2 \mathrm{~L})^{4}$. Importantly, transgene integration at $40 \mathrm{D}$ is associated with dominant phenotypes when crossed to some Gal4 drivers ${ }^{4}$.

The Hippo pathway constitutes an essential and evolutionarily conserved growth-regulatory network ${ }^{5-10}$. RNAi screens in D. melanogaster have facilitated the discovery of many new components of the Hippo pathway ${ }^{11-17}$. The key downstream component of the Hippo pathway is Yorkie (Yki), a growth-promoting transcriptional co-activator ${ }^{18}$. Expression of an activated form of $\mathrm{Yki}\left(\mathrm{Yki}^{\mathrm{S} 168 \mathrm{~A}}\right)$ in the eyes leads to overgrowth, which we and others have used as a sensitized background to screen for new Hippo pathway proteins ${ }^{11-14}$.
In this study, we show that transgene insertion at the 40D locus in a subset of VDRC KK RNAi lines is associated with aberrant enhancement of Hippo pathway phenotypes. The effect is not due to knockdown of intended target genes by RNAi. Rather, our data suggest that the effect is due to ectopic UAS-driven expression of the Tiptop transcription factor. Ectopic tiptop (tio) expression linked to a subset of VDRC KK RNAi lines causes deleterious phenotypes in different organs, as evidenced by the fact that broad expression causes organism lethality ${ }^{4}$. Of relevance to the Drosophila research community, we describe a D. melanogaster strain that will allow researchers to test, in a single cross, whether their genetic screen of interest will be affected by ectopic tio expression.

\section{Results and Discussion}

25\% of VDRC KK RNAi lines enhance Yki-mediated overgrowth. To screen for new components of the Hippo pathway, we drove expression of an activated form of Yki $\left(\mathrm{Yki}^{\mathrm{S} 168 \mathrm{~A}}\right)^{19}$ in the eye using GMR-Gal4 and crossed this strain to RNAi lines from the VDRC KK library. We then scored for modulation of the Yki-induced eye overgrowth phenotype. We observed that a large number of VDRC KK RNAi lines enhanced Yki-induced eye overgrowth, particularly in the posterior part of the eye, which we scored as enhancers (Fig. 1a-c). However, in experiments analysing a subset of these enhancers with independent RNAi lines (such as VDRC GD, TRiP or NIG RNAi lines) or mutant alleles, the original phenotype was not
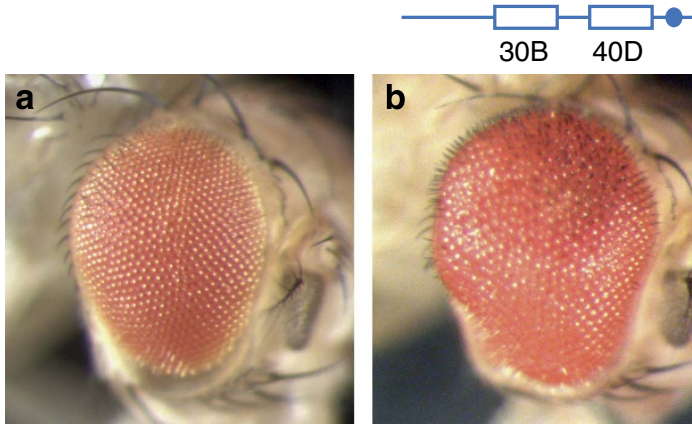

Control

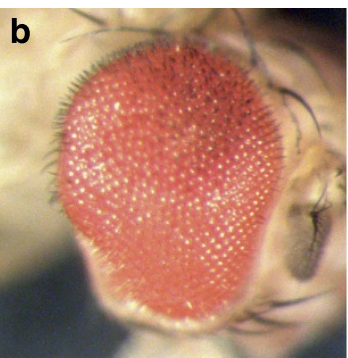

$y k i S 168 A$
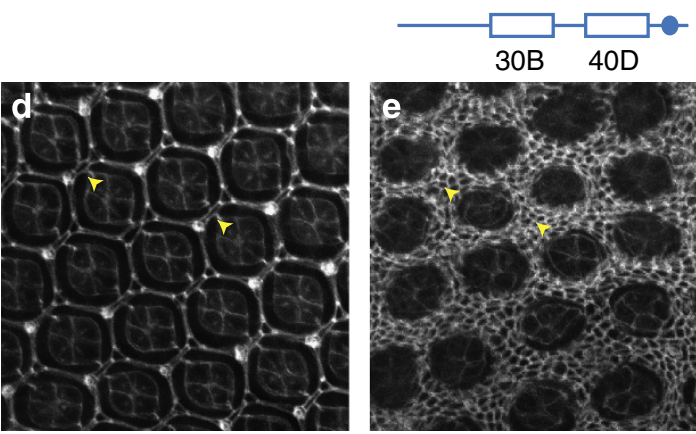

Control

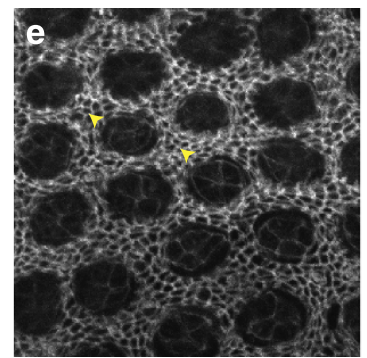

$y k i^{S 168 A}$
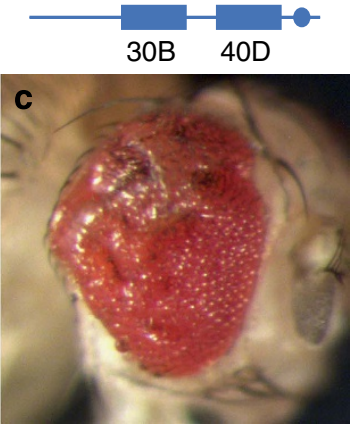

$y k i^{S 168 A}, \mathrm{KK}$
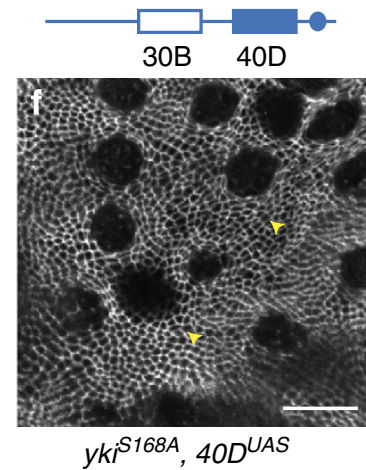

Empty transgene landing site

Transgene landing site with integrated transgene

Figure 1 | Select VDRC KK RNAi lines enhance Yorkie-induced eye overgrowth. (a) Wildtype eyes ( $F_{1}$ flies of VDRC genetic background crossed to GMR-Gal4, UAS-EYFP). (b,c) Adult eye phenotypes of $F_{1}$ flies carrying GMR-Gal4, UAS-yki ${ }^{S 168 A_{-}}$-YFP transgenes crossed to VDRC genetic background (b) or VDRC line 105838 (targeting Caf1) (c). (d-f) Posterior area of pupal eyes of wildtype (d) and $F_{1}$ flies carrying GMR-Gal4, UAS-yki ${ }^{5168 A}-$ YFP transgenes crossed to VDRC genetic background (e) or a recombinant harbouring Gal4-responsive UAS repeats but no functional shRNA-coding sequence at $40 D\left(' 40 D^{U A S}\right)(\mathbf{f})$. Pupal eyes were fixed and stained with Discs large antibody to highlight individual cells. Yellow arrowheads indicate interommatidial cells. Scale bar, $20 \mu \mathrm{m}$. 
recapitulated (Supplementary Fig. 1), suggesting that it was not caused by depletion of the intended target gene.

Based on the findings of Green et al. ${ }^{4}$, we genotyped these KK RNAi lines by PCR to determine their integration site occupancy. We observed that most KK RNAi lines that enhanced Yki-driven eye overgrowth possessed transgenes at both $40 \mathrm{D}$ and $30 \mathrm{~B}$ on chromosome arm 2L (Fig. 2a-g). Notably, the percentage of KK RNAi lines we scored as enhancers (38 out of 150,25\%) is very similar to the percentage of KK RNAi lines reported by Green et al. ${ }^{4}$, to contain insertions at both $40 \mathrm{D}$ and 30B $(9 / 39,23 \%)$. We separated the two insertion sites in these lines by meiotic recombination and observed that enhancement of Yki-mediated eye overgrowth co-segregated with transgene insertion at $40 \mathrm{D}$
(Fig. 2a-g). This result strongly suggests that the observed phenotypic enhancement is an artefact associated with transgene insertion at the 40D locus. However, it did not formally exclude the possible contribution of knockdown of intended target genes.

To investigate this further, we made use of a KK RNAi line that harbours Gal4-responsive UAS repeats but no functional short hairpin RNA (shRNA) coding sequence at 40D (E. Green, personal communication, see also Methods). We used meiotic recombination to remove the transgene insertion at $30 \mathrm{~B}$. Using the resulting ' $40 D^{U A S}$ ' line, we tested whether enhancement of Yki-mediated overgrowth was caused by UAS-dependent overexpression of a gene at $40 \mathrm{D}$ or expression of the shRNA. The $40 D^{U A S}$ line enhanced Yki-mediated overgrowth (Fig. 2j) and

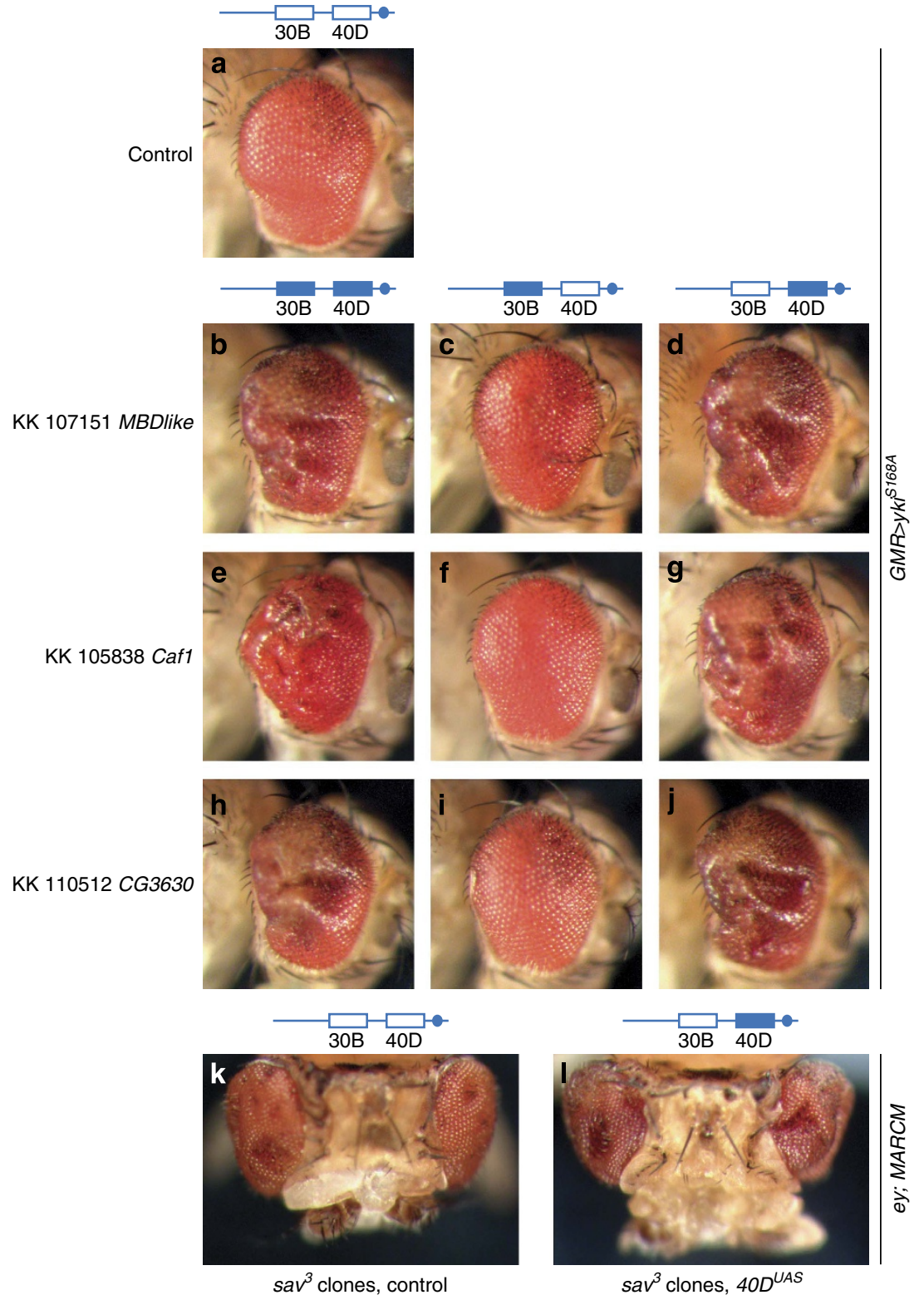

Figure 2 | Enhancement of Yorkie-induced eye overgrowth by VDRC KK RNAi lines co-segregates with insertion at 40D and is independent of knockdown of intended target genes. (a-j) Adult eye phenotypes of $F_{1}$ flies carrying GMR-Gal4, UAS-yki ${ }^{S 168 A_{-}}$YFP transgenes crossed to VDRC genetic background (a), VDRC line 107151 (MBD-like) with double insertion (b), single insertion at 30B (c) and 40D (d), VDRC line 105838 (Caf1) with double insertion (e), single insertion at 30B (f) and 40D (g), VDRC line 110512 (CG3630) with double insertion (h), single insertion at 30B (i) and 40D. The transgene insertion at 4OD in line 110512 does not carry a functional hairpin-coding sequence, hence it is called ' $40 D^{\text {UAS' }}$ throughout this manuscript. (j). $(\mathbf{k}, \mathbf{I})$ Eye phenotypes of flies harbouring $s a v^{3}$ clones expressing Gal4 in the eye combined with the VDRC genetic background (k) and $40 D^{U A S}(\mathbf{I})$. Sav is an upstream negative regulator of $\mathrm{Yki}$, therefore the $\mathrm{sa}^{3}$ mutation causes $\mathrm{Yki}$ hyperactivation. Clones were generated using the Mosaic analysis with a repressible cell marker (MARCM) system under the control of the eyeless (ey) promoter. 
caused a substantial increase in interommatidial cells (Fig. 1f), considered a classic Hippo pathway mutant phenotype ${ }^{20}$.

By contrast, in three independent lines expressing shRNA hairpins for MBD-like, Caf1 and CG3630 from the insertion site at $30 \mathrm{~B}$, but not $40 \mathrm{D}$, we observed no modification of Yki-driven eye overgrowth (Fig. 2a-j). Therefore, enhancement of Yki-mediated eye overgrowth was caused by transgene integration at $40 \mathrm{D}$, rather than $\mathrm{RNAi}$-mediated depletion of target genes.

To assess this result in an independent genetic context, we generated flies with clones of eye tissue mutant for salvador (sav), a member of the core kinase cassette of the Hippo pathway ${ }^{20,21}$. Loss of function of sav leads to Yki hyperactivation ${ }^{18}$ and overgrowth $^{20,21}$. The eye overgrowth phenotype of flies harbouring sav mutant clones expressing Gal4 combined with $40 D^{U A S}$ was enhanced to a similar degree as we observed when $Y k i^{S 168 A}$ was overexpressed (Fig. $2 \mathrm{k}, \mathrm{l}$ ). This confirms that transgene insertion at $40 \mathrm{D}$ in KK RNAi lines enhances Yki-mediated tissue overgrowth irrespective of the mechanism by which Yki is activated.

Ectopic tiptop expression in a subset of VDRC KK RNAi lines. Green et al. ${ }^{4}$ suggested that the dominant effects caused by transgene insertion at $40 \mathrm{D}$ were caused by overexpression of the tio gene, given that KK RNAi lines were engineered to be inserted at a locus at $40 \mathrm{D}$ that is located in the tio $5^{\prime}$ untranslated region. This hypothesis gained our attention, because Yki was reported to regulate eye growth, at least in part, together with Teashirt (Tsh) ${ }^{22}$, a functionally redundant tio paralogue ${ }^{23}$. Indeed, an independent
UAS-regulated tio transgene ${ }^{24}$ also enhanced Yki-mediated eye overgrowth (Fig. 3a,b). Moreover, tio mRNA expression was increased approximately twofold in eye imaginal discs from larvae combining eye-specific Gal4 expression with the aforementioned $40 D^{U A S}$ line (Fig. 3c). Taken together, these findings strongly suggest that ectopic tio expression caused by insertion of UAScarrying transgenes at 40D in some KK RNAi lines is responsible for enhancement of Yki-mediated eye overgrowth. The $40 D^{U A S}$ line or UAS-tio did not enhance eye growth in flies expressing EYFP (control), dMyc or insulin receptor ( $\mathrm{InR}$ ), although high tio overexpression caused patterning defects in the posterior part of the eye (Supplementary Fig. 2). Therefore, ectopic tio expression specifically modulates Hippo pathway-mediated tissue growth, at least when these growth pathways are modulated in the developing eye using GMR-Gal4.

An important validation of newly identified Hippo pathway components is the demonstration that they regulate expression of Yki target genes such as bantam (ban). We found that, although high tio overexpression induced ban expression, the $40 D^{U A S}$ line did not (Fig. 3d,e). This shows that, like tsh, ectopic tio expression can drive expression of Yki target genes. Changes in ban expression due to ectopic tio expression in $\mathrm{KK}$ lines were probably beyond the limits of detection in our experimental setting given that we observed clear effects on tissue growth.

Resources to enhance the use of the VDRC KK RNAi library. To provide a resource for other researchers, we genotyped a subset of commonly used KK RNAi lines directed against Hippo pathway genes and identified several that have transgene
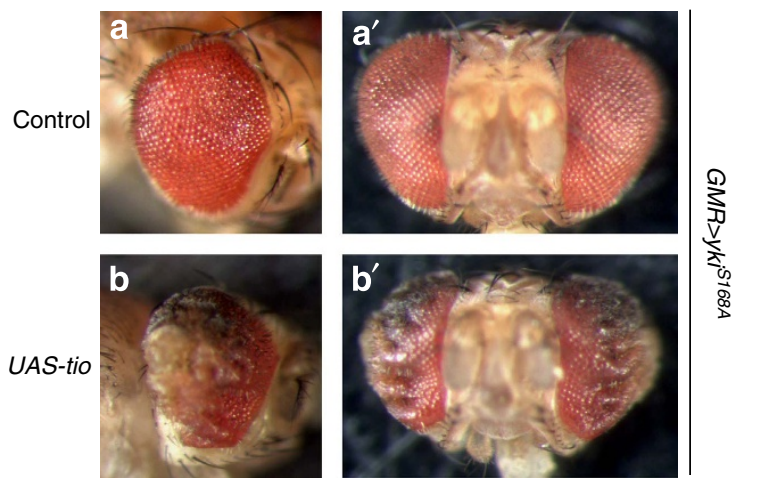

C
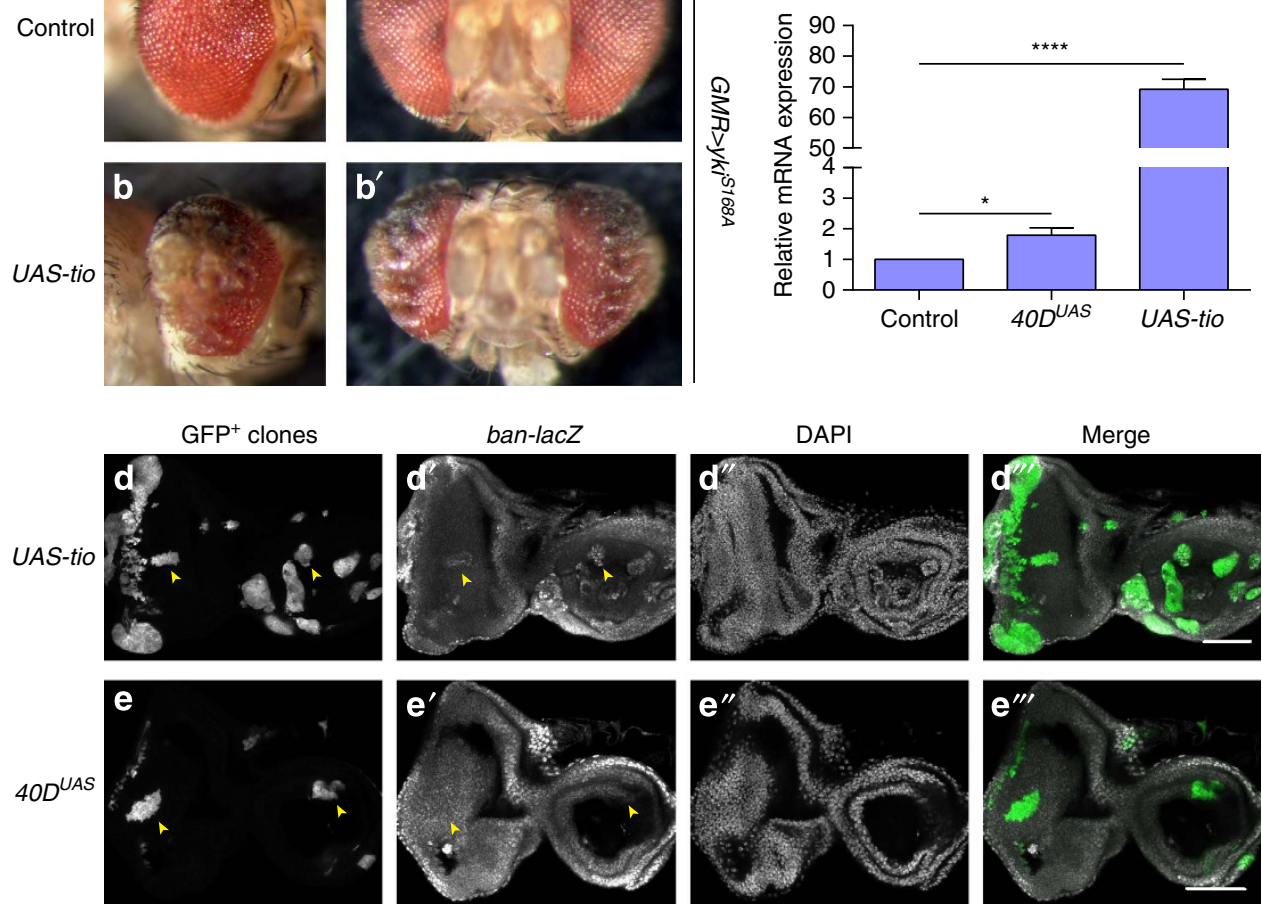

Figure 3 | Overexpression of tiptop enhances Yorkie-induced eye overgrowth and induces bantam expression. (a,b) Adult eye phenotypes of $F_{1}$ flies carrying GMR-Gal4, UAS-yki ${ }^{5168 A}$-YFP transgenes crossed to UAS control (a) and UAS-tio (b). (c) Tio mRNA expression in eye imaginal discs of $\mathrm{F}_{1}$ flies carrying GMR-Gal4 crossed to VDRC genetic background, 4ODUAS and UAS-tio (positive control). QPCR was performed using two different primer sets probing tio mRNA expression, and normalized against $A c t 5 c$ and Rp49 mRNA-loading controls, yielding identical results (only tio primer set 1 and Act5c results shown). Average results from three independent experiments are shown. Error bars indicate s.e.m. ${ }^{\star} P<0.05$, ${ }^{\star \star \star *} P<0.0001$, unpaired two-tailed $t$-test. (d,e) Actin-Flp-out clones (yellow arrowheads) expressing Gal4 in combination with UAS-tio (d) or 40D UAS (e) in larval eye imaginal discs expressing the ban-lacZ reporter. Scale bars, $50 \mu \mathrm{m}$. 

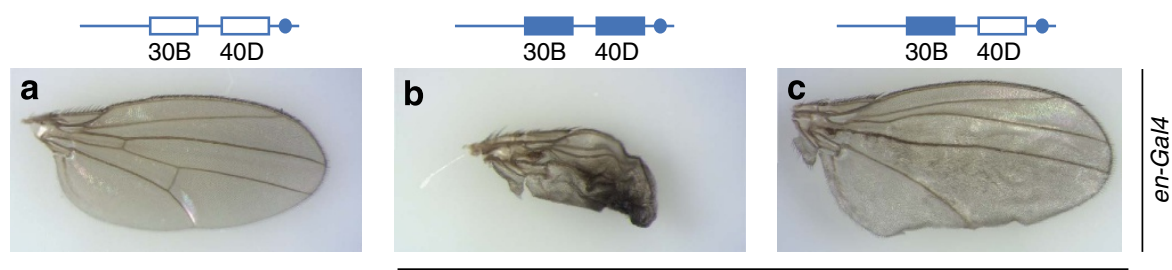

Control

KK 106174 wts
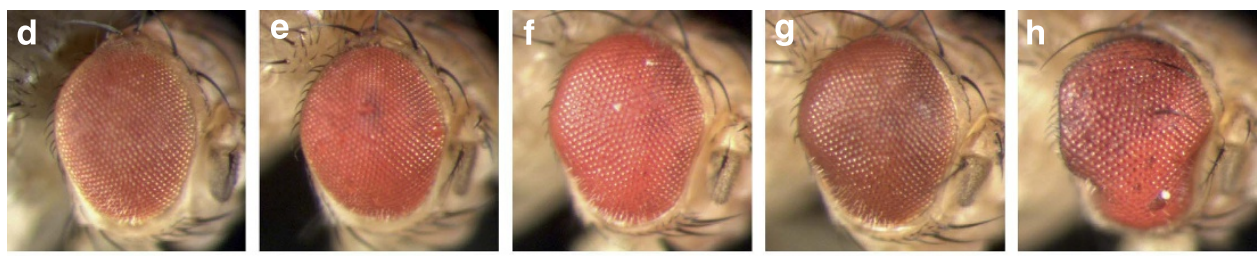

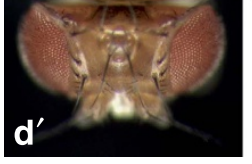

Control

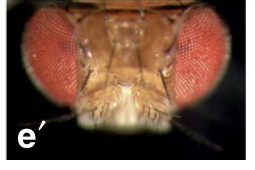

TRiP BL27662

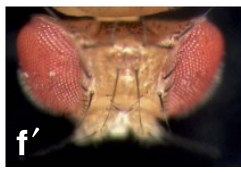

NIG 12072R-1

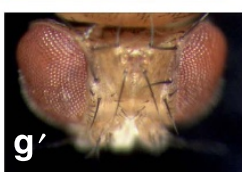

VDRC GD9928

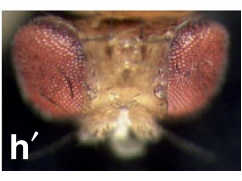

VDRC KK 106174 30B only

GMR>wts RNAi

Figure 4 | Separation of insertion sites in the Warts KK RNAi line reveals potential for false-negative screening results and shows the power of KK RNAi lines if the 40D integration is removed. (a-c) Wings of control flies (a) and $\mathrm{F}_{1}$ flies carrying en-Gal4 crossed to VDRC line 106174 (wts) with double insertion (b) or single insertion at $30 \mathrm{~B}(\mathbf{c})$. Flies were reared at $18^{\circ} \mathrm{C}$. (d-h) Adult eye phenotypes of $\mathrm{F}_{1}$ flies carrying $G M R$-Gal4 crossed to VDRC genetic background (d), and wts RNAi lines Bloomington TRiP 27662 (e), NIG 12072R-1 (f), VDRC GD line 9928 (g) and VDRC KK line 106174, insertion at 30B only (h).

insertions at 40D (Supplementary Table 1 and Supplementary Fig. 3). Separation of insertion sites of the KK RNAi line targeting the Hippo pathway kinase Warts (Wts) revealed two important findings: first, wing overgrowth caused by Wts knockdown was masked by the non-inflating wing phenotype caused by transgene insertion at $40 \mathrm{D}^{4}$ (Fig. 4a-c); second, eye-specific Wts knockdown in a line that harbours a transgene insertion at $30 \mathrm{~B}$ only, led to more dramatic eye overgrowth than other publicly available Wts RNAi lines (Fig. 4d-h), illustrating the potential power of KK RNAi lines when free of transgene insertions at 40D.

Finally, we crossed the $40 D^{U A S}$ line to a collection of Gal4 drivers and assessed overt phenotypes in adult progeny (Supplementary Table 2). In particular, crosses to wing drivers caused substantial malformations, whereas eye drivers did not. Some wing drivers led to the non-inflating wing phenotype as described for elav-gal4 $4^{155}$ (ref. 4) (Supplementary Fig. 4b), whereas others caused wing shape changes (Supplementary Fig. 4d). Broad expression of the $40 D^{U A S}$ line caused organism lethality.

In conclusion, some KK RNAi lines can give false-positive results in eye-based screens for Hippo pathway genes and can also cloud growth screens performed in tissues such as the wing. The VDRC KK RNAi library has proven to be an extremely powerful resource for exploring gene function. Importantly, the $40 D^{U A S}$ flies we have generated will allow researchers to test, in a single cross (Supplementary Fig. 4e), whether their screen of interest will be affected by ectopic tio expression that is associated with a subset of KK RNAi lines.

\section{Methods}

Drosophila genetics. VDRC KK and GD RNAi lines were from VDRC, Austria. Other RNAi lines were from NIG, Japan or Bloomington, USA. Drosophila KK RNAi strains carrying a single transgene insertion were generated by meiotic recombination and their genotype confirmed by $\mathrm{PCR}^{4}$.

Male flies from the KK RNAi library, recombinant strains and $w ;$ UAS-tio ${ }^{24}$ were crossed to $w ;$; GMR-Gal4, UAS- $y k i S 168 A_{-Y F P} / T M 6 B^{11}, w ;$; GMR-Gal4, UAS-EYFP/TM6B, w; GMR-Gal4 or $w$; en-Gal4 virgin female flies.
For the eyFlp, MARCM experiment, genotypes were:

$y$ w eyFlp, UAS-green fluorescent protein (UAS-GFP); GD60100/ +; tub-Gal4 FRT82B tub-Gal80/FRT82B sav

y w eyFlp, UAS-GFP; 40D UAS/+; tub-Gal4 FRT82B tub-Gal80/FRT82B sav ${ }^{3}$

For the Flp-out experiment, genotypes were:

$y$ w hsFLP;40DUAS/ + ; act >CD2> GAL4, UAS-GFP/ ban-lacZ

$y$ w hsFLP;; act $>C D 2>$ GAL4, UAS-GFP/ UAS-tio-FL, ban-lacZ

The ban-lacZ allele is described in Flybase (P\{lacW\}banL1170a) and was from Bloomington.

At least 30 adult organs (eyes and wings) of 1-2-day-old females were assessed.

See Supplementary Methods for genotypes presented in Supplementary Information.

PCR and sequencing. Primers were designed using Primer3. Genomic DNA was isolated by crushing flies in lysis buffer $(0.2 \mathrm{M}$ Sucrose, $0.1 \mathrm{M}$ Tris, $\mathrm{pH} 9.2,50 \mathrm{mM}$ EDTA, $0.5 \%$ SDS), $10 \mathrm{~min}$ incubation at $65^{\circ} \mathrm{C}$, addition of $1 \mathrm{M} \mathrm{KAc}, 15 \mathrm{~min}$ incubation on ice, phenol-chloroform extraction and isopropanol precipitation. KK insertion site occupancy was determined as in ref. 4. Briefly, occupancy of the transgene insertion site at 40D was determined in multiplex PCR using the following primers:

C_Genomic_F 5'-GCCCACTGTCAGCTCTCAAC- $3^{\prime}$

pKC26_R 5'-TGTAAAACGACGGCCAGT-3'

pKC43_R 5'-TCGCTCGTTGCAGAATAGTCC-3'

PCR using these primers yields a $\sim 450$ bp product in the case of an insertion, or a $\sim 1,050$ bp product in the case of an empty insertion site, at $40 \mathrm{D}$.

For the insertion at 30B, the pKC26_R and pKC43_R primers were multiplexed with the following primer:

NC_Genomic_F 5'-GCTGGCGAACTGTCAATCAC-3'

PCR using these primers results in a $\sim 600$ bp product in the case of an insertion, and a $\sim 1,200 \mathrm{bp}$ product in the case of an empty insertion site, at 30B.

PCR was performed using Qiagen Taq PCR master mix in an Eppendorf thermal cycler with the following programme: $95^{\circ} \mathrm{C}, 120 \mathrm{~s}$ initial denaturation, 30 cycle reaction $\left(95^{\circ} \mathrm{C} 15 \mathrm{~s}\right.$ denaturation, $51^{\circ} \mathrm{C} 15 \mathrm{~s}$ annealing, $72^{\circ} \mathrm{C} 80 \mathrm{~s}$ extension), and a final $72{ }^{\circ} \mathrm{C}, 120 \mathrm{~s}$ extension. Primers to detect whether pKC26 was filled were:

KK_UAS_f $5^{\prime}$-TAGGCACCCCAGGCTTTAC- $3^{\prime}$

KK_UAS_r 5' $5^{\prime}$-TTTGTCCAATTATGTCACACCAC-3'

PCR was performed with the following programme: $95^{\circ} \mathrm{C}, 120 \mathrm{~s}$ initial denaturation, 30 cycle reaction $\left(95^{\circ} \mathrm{C} 15 \mathrm{~s}\right.$ denaturation, $50^{\circ} \mathrm{C} 15 \mathrm{~s}$ annealing, $72^{\circ} \mathrm{C} 45 \mathrm{~s}$ extension), and a final $72^{\circ} \mathrm{C}, 120 \mathrm{~s}$ extension. These primers amplify a 700 bp product within empty pKC26, and do not amplify if a RNA

hairpin-encoding sequence is inserted. PCR was performed using KK transformant ID 110512 genomic DNA, and the product cloned and sequenced. The sequence 
inserted is $5^{\prime}$-TAGGTCTTTCCCTTGGCGGGGCTTGACCTATCTAGA-3' . Blast searches using this sequence against Drosophila RefSeq RNA, genome and the $\mathrm{nr}$ database did not recover any meaningful matches.

Quantitative real-time PCR. Per condition, 30 eye-antennal discs were dissected from late L3 larvae from crosses of $w$; GMR-Gal4 females to males of the indicated genotypes, and lysed in Trizol reagent (Life Technologies). RNA isolation was performed according to manufacturer's instructions. RNA $(0.5 \mu \mathrm{g})$ was treated with DNAse (Promega) followed by addition of oligo-dT and retrotranscription using SuperScript III (Promega). Quantitative PCR was performed using Sybr Green Fast (Life Technologies) on a StepOnePlus system (Life Technologies). Quantitative PCR was performed using two different primer sets probing tio mRNA expression, and normalized against $A c t 5 c$ and Rp49 mRNA-loading controls, yielding identical results (only tio primer set 1 and $A c t 5 c$ results shown).

The following primers were used:

qTio_fwd1_2430 5'-TGGAGAACAGCGGCAATAAC- ${ }^{\prime}$

qTio_rev1_2557 5'-ATCCGTGGTCTCGCACAG-3'

qTio_fwd2_2388 $5^{\prime}$-GTCTACTGGCGTTGAACTCC-3'

qTio_rev2_2529 5'-GCAGCTAGAGGATGGGCC-3'

qActin_5c_f $5^{\prime}$-CTCCAAGCAGGAGTACGACGAG-3'

qActin_5c_r 5'-CTTCCACCACTCGCACTTGC-3'

qRpL32 f $5^{\prime}$-CGCACCAAGCACTTCATCCG-3'

qRpL32_r 5'-GCACGTTGTGCACCAGGAAC-3'

Microscopy. Pupal eyes were dissected in PBS from staged pupae $46 \mathrm{~h}$ after puparium formation. Flp-out clones were generated by $30 \mathrm{~min}$ of heat shock $\left(37^{\circ} \mathrm{C}\right)$, $48 \mathrm{~h}$ prior to dissection. L3 larvae were dissected in PBS. Samples were fixed for $20 \mathrm{~min}$ in 4\% paraformaldehyde (Electron Microscopy Sciences \#15710), rinsed once and washed three times for $10 \mathrm{~min}$ in $0.3 \%$ PBT. Samples were then blocked for $30 \mathrm{~min}$ in $0.1 \%$ PBT with $10 \%$ NGS and incubated for $4 \mathrm{~h}$ at $4{ }^{\circ} \mathrm{C}$ with primary antibodies in $0.1 \%$ PBT with $10 \%$ NGS. Samples were then washed three times for $12 \mathrm{~min}$ in $0.3 \%$ PBT, before secondary antibodies were added in $0.1 \%$ PBT and $10 \%$ NGS and incubated overnight at $4{ }^{\circ} \mathrm{C}$. Samples were then washed three times for $12 \mathrm{~min}$ in $0.3 \%$ PBT, DAPI was added with the second wash. Samples were mounted in $90 \%$ glycerol in PBS and imaged. Confocal images were acquired using a Nikon $\mathrm{C} 2$ confocal microscope using a $\times 20$ (numerical aperture $(\mathrm{NA})=0.75)$ or $40 \times$ ( $\mathrm{NA}=1.00$, oil immersion) objective and processed using Fiji (Image)).

Primary antibodies used were $\beta$-galactosidase (mouse, 1:100, Sigma G4644) and Discs large (mouse, 1:50, Developmental Studies Hybridoma Bank \#2A1). Secondary antibody was anti-mouse 555 (goat, 1:500, Molecular Probes).

Statistics. No statistical test was used to predetermine sample size in any of the experiments. For animal experiments, at least 30 animals were assessed in each experiment. No samples or animals were excluded from the analysis in any of the experiments. The experiments were not randomized. Investigators were not blinded to allocation during experiments or outcome assessment.

In Fig. 3c, an unpaired two-tailed $t$-test was used. The data met the assumptions of the test. S.e.m. is indicated. The variance is similar between the groups that are being statistically compared.

\section{References}

1. Dietzl, G. et al. A genome-wide transgenic RNAi library for conditional gene inactivation in Drosophila. Nature 448, 151-156 (2007).

2. Mohr, S. E., Smith, J. A., Shamu, C. E., Neumuller, R. A. \& Perrimon, N. RNAi screening comes of age: improved techniques and complementary approaches. Nat. Rev. Mol. Cell Biol. 15, 591-600 (2014).

3. Chen, K. F. \& Crowther, D. C. Functional genomics in Drosophila models of human disease. Brief. Funct. Genomics 11, 405-415 (2012).

4. Green, E. W., Fedele, G., Giorgini, F. \& Kyriacou, C. P. A Drosophila RNAi collection is subject to dominant phenotypic effects. Nat. Methods 11, 222-223 (2014)

5. Harvey, K. F., Zhang, X. \& Thomas, D. M. The Hippo pathway and human cancer. Nat. Rev. Cancer 13, 246-257 (2013).

6. Pan, D. The hippo signaling pathway in development and cancer. Dev. Cell 19, 491-505 (2010).

7. Halder, G. \& Johnson, R. L. Hippo signaling: growth control and beyond Development 138, 9-22 (2011).

8. Irvine, K. D. Integration of intercellular signaling through the Hippo pathway. Semin. Cell Dev. Biol. 23, 812-817 (2012).

9. Gaspar, P. \& Tapon, N. Sensing the local environment: actin architecture and Hippo signalling. Curr. Opin. Cell Biol. 31, 74-83 (2014).

10. Enderle, L. \& McNeill, H. Hippo gains weight: added insights and complexity to pathway control. Sci. Signal. 6, re7 (2013).

11. Poon, C. L., Lin, J. I., Zhang, X. \& Harvey, K. F. The sterile 20-like kinase Tao-1 controls tissue growth by regulating the Salvador-Warts-Hippo pathway. Dev. Cell 21, 896-906 (2011).
12. Poon, C. L., Zhang, X., Lin, J. I., Manning, S. A. \& Harvey, K. F. Homeodomain-interacting protein kinase regulates Hippo pathway-dependent tissue growth. Curr Biol. 22, 1587-1594 (2012).

13. Yue, T., Tian, A. \& Jiang, J. The cell adhesion molecule echinoid functions as a tumor suppressor and upstream regulator of the Hippo signaling pathway. Dev. Cell 22, 255-267 (2012).

14. Zhang, H. et al. SCF(Slmb) E3 ligase-mediated degradation of Expanded is inhibited by the Hippo pathway in Drosophila. Cell. Res. 25, 93-109 (2015).

15. Deng, H. et al. Spectrin regulates Hippo signaling by modulating cortical actomyosin activity. eLife 4, e06567 (2015).

16. Genevet, A., Wehr, M. C., Brain, R., Thompson, B. J. \& Tapon, N. Kibra is a regulator of the Salvador/Warts/Hippo signaling network. Dev. Cell 18, 300-308 (2010).

17. Rauskolb, C., Pan, G., Reddy, B. V., Oh, H. \& Irvine, K. D. Zyxin links fat signaling to the hippo pathway. PLoS Biol. 9, e1000624 (2011).

18. Huang, J., Wu, S., Barrera, J., Matthews, K. \& Pan, D. The Hippo signaling pathway coordinately regulates cell proliferation and apoptosis by inactivating Yorkie, the Drosophila Homolog of YAP. Cell 122, 421-434 (2005).

19. Zhang, X., Milton, C. C., Humbert, P. O. \& Harvey, K. F. Transcriptional output of the Salvador/warts/hippo pathway is controlled in distinct fashions in Drosophila melanogaster and mammalian cell lines. Cancer Res. 69, 6033-6041 (2009).

20. Tapon, N. et al. salvador Promotes both cell cycle exit and apoptosis in Drosophila and is mutated in human cancer cell lines. Cell 110, 467-478 (2002).

21. Kango-Singh, M. et al. Shar-pei mediates cell proliferation arrest during imaginal disc growth in Drosophila. Development 129, 5719-5730 (2002).

22. Peng, H. W., Slattery, M. \& Mann, R. S. Transcription factor choice in the Hippo signaling pathway: homothorax and yorkie regulation of the microRNA bantam in the progenitor domain of the Drosophila eye imaginal disc. Genes Dev. 23, 2307-2319 (2009)

23. Bessa, J., Carmona, L. \& Casares, F. Zinc-finger paralogues tsh and tio are functionally equivalent during imaginal development in Drosophila and maintain their expression levels through auto- and cross-negative feedback loops. Dev. Dyn. 238, 19-28 (2009).

24. Denholm, B. et al. The tiptop/teashirt genes regulate cell differentiation and renal physiology in Drosophila. Development 140, 1100-1110 (2013).

\section{Acknowledgements}

We thank Edward Green for helpful comments, Nan Hu and Barry Denholm for UAS-tio flies and members of the Harvey laboratory and Kate Sutherland for discussions and critical reading of the manuscript. We thank the Vienna Drosophila RNAi Center, the Bloomington Drosophila Stock Centre, the National Institute of Genetics, Japan, the Australian Drosophila Research Support Facility (www.ozdros.com), and the Developmental Studies Hybridoma Bank for fly stocks and antibodies. K.F.H is a National Health and Medical Research Council Senior Research Fellow. S.A.M. was supported by a 2012 Australian Postgraduate Award scholarship. A.K. was supported by a Cancer Council Victoria Vacation Studentship. This research was supported by a Project Grant from the National Health and Medical Research Council of Australia.

\section{Author contributions}

J.H.A.V. designed the study. All authors contributed to experiments. J.H.A.V. and K.F.H. wrote the manuscript, with help from S.A.M. All authors read and approved the final version of the manuscript.

\section{Additional information}

Supplementary Information accompanies this paper at http://www.nature.com/ naturecommunications

Competing financial interests: The authors declare no competing financial interests.

Reprints and permission information is available online at http://npg.nature.com/ reprintsandpermissions/

How to cite this article: Vissers, J. H. A. et al. A Drosophila RNAi library modulates Hippo pathway-dependent tissue growth. Nat. Commun. 7:10368 doi: 10.1038/ncomms10368 (2016)

This work is licensed under a Creative Commons Attribution 4.0 International License. The images or other third party material in this article are included in the article's Creative Commons license, unless indicated otherwise in the credit line; if the material is not included under the Creative Commons license, users will need to obtain permission from the license holder to reproduce the material To view a copy of this license, visit http://creativecommons.org/licenses/by/4.0/ 